\title{
Insights into the mechanism of the effects of rhizosphere microorganisms on the quality of authentic Angelica sinensis under different soil microenvironments
}

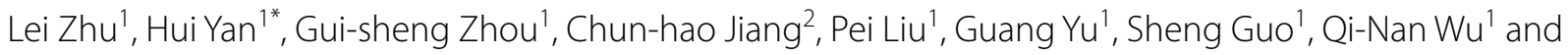
Jin-ao Duan ${ }^{1 *}$

\begin{abstract}
Background: Angelica sinensis (Oliv.) Diels (A. sinensis) is a Chinese herb grown in different geographical locations. It contains numerous active components with therapeutic value. Rhizosphere microbiomes affect various aspects of plant performance, such as nutrient acquisition, growth and development and plant diseases resistance. So far, few studies have investigated how the microbiome effects level of active components of $A$. sinensis. This study investigated whether changes in rhizosphere microbial communities and metabolites of $A$. sinensis vary with the soil microenvironment. Soils from the two main A. sinensis-producing areas, Gansu and Yunnan Province, were used to conduct pot experiments. The soil samples were divided into two parts, one part was sterilized and the other was unsterilized planting with the seedling variety of Gansu danggui 90-01. All seedlings were allowed to grow for 180 days. At the end of the experiment, radix A. sinensis were collected and used to characterize growth targets and chemical compositions. Rhizosphere soils were subjected to microbial analyses.
\end{abstract}

Results: Changes in metabolic profiles and rhizosphere microbial communities of $A$. sinensis grown under different soil microenvironments were similar. The GN (Gansu non-sterilized), YN (Yunnan non-sterilized), GS (Gansu sterilized), and YS (Yunnan sterilized) groups were significantly separated. Notably, antagonistic bacteria such as Sphingomonas, Pseudomonas, Lysobacter, Pseudoxanthomonas, etc. were significantly $(p<0.05)$ enriched in Gansu soil compared with Yunnan soil. Moreover, senkyunolide I and ligustilide dimers which were enriched in GS group were strongly positively correlated with Pseudomonas parafulva; organic acids (including chlorogenic acid, dicaffeoylquinic acid and 5-feruloylquinic acid) and their ester coniferyl ferulate which were enriched in YS Group were positively associated with Gemmatimonadetes bacterium WY71 and Mucilaginibater sp., respectively.

Conclusions: The soil microenvironment influences growth and level/type of active components in A. sinensis. Further studies should explore the functional features of quality-related bacteria, identify the key response genes and

\footnotetext{
*Correspondence: glory-yan@163.com; dja@njucm.edu.cn

${ }^{1}$ National and Local Collaborative Engineering Center of Chinese Medicinal Resources Industrialization and Formulae Innovative Medicine, Jiangsu Collaborative Innovation Center of Chinese Medicinal Resources Industrialization, State Administration of Traditional Chinese Medicine Key Laboratory of Chinese Medicinal Resources Recycling Utilization, Nanjing University of Chinese Medicine, Nanjing, China

Full list of author information is available at the end of the article
}

(C) The Author(s) 2021. Open Access This article is licensed under a Creative Commons Attribution 4.0 International License, which permits use, sharing, adaptation, distribution and reproduction in any medium or format, as long as you give appropriate credit to the original author(s) and the source, provide a link to the Creative Commons licence, and indicate if changes were made. The images or other third party material in this article are included in the article's Creative Commons licence, unless indicated otherwise in a credit line to the material. If material is not included in the article's Creative Commons licence and your intended use is not permitted by statutory regulation or exceeds the permitted use, you will need to obtain permission directly from the copyright holder. To view a copy of this licence, visit http://creativecommons.org/licenses/by/4.0/. The Creative Commons Public Domain Dedication waiver (http://creativeco mmons.org/publicdomain/zero/1.0/) applies to the data made available in this article, unless otherwise stated in a credit line to the data. 
clarify the interactions between genes and soil environments. This will reveal the mechanisms that determine the quality formation of genuine $A$. sinensis.

Keywords: Angelica sinensis, Rhizosphere microorganism, Metabolomics, quality, Correlation

\section{Background}

Geo-authentic Chinese medicinal materials are traditional Chinese medicine (TCM) selected through longterm clinical practice. They exhibit satisfactory curative effect, stable quality, and high profile. In particular, the radix $A$. sinensis possess traditional efficacies; for instance, it nourishes the blood, promotes blood circulation, regulates menstruation, and relieves pain. It was first recommended as a therapeutic option for gynecological diseases [1] and is currently utilized for general protection of the heart, immune booster, anti-arrhythmia, and anti-atherosclerosis, etc. [2]. In China, A. sinensis is largely produced in Gansu, Yunnan, Qinghai, and other places. Min County of Gansu Province has long been acknowledged as its traditional geoherb region with a cool and humid climate, abundant rainfall, mostly fertile cinnamon and black soil, which are ideal for $A$. sinensis growth. Terrestrial plants and soil microorganisms mutually benefit through strong interactions. Plants provide habitats and reliable carbon and energy sources for soil microorganisms, which directly or indirectly contribute to the yield and quality of the host plants. Also, they boost the hosts' resistance by improving soil mineral nutrition, production of phytohormones, degradation of phytotoxic compounds, inhibition of soil-borne pathogens, etc. [3, 4]. Numerous reports indicate that plant species, growth, and development, host genotypes, geographical location, are the main drivers of rhizosphere microbial diversity [5-7]. Notably, geographical position and cultivation practice are the key factors that determine microbiome variation in field conditions; however, under controlled greenhouse conditions, the microbial composition varies with soil source and genotype [6]. Similar results were reported in a large-scale longitudinal field study that compared changes in microbial communities across 27 maize inbred lines planted in three fields [7]. Contrarily, plant genetic effects had a remarkable impact on the rhizosphere microbiome, surpassing geographic location, plant age, and climatic events.

Compelling evidence indicates that plant-microbe interactions are vital for healthy plant growth [8]. Currently, the key interest to explore genuine TCMs is mainly geared on soil properties and rhizosphere microorganisms. Important breakthroughs have been made in the growth and yield improvement of famous medicinal materials, including Panax ginseng, Rehmannia glutinosa, and Panax notoginseng, etc. Changes in rhizosphere microbial communities and accumulation of toxic substances are greatly associated with the replanting failure of TCMs. For instance, reports show that soil-borne pathogen, Fusarium flourishes as plants grow, whereas antagonistic bacterium Pseudomonas and Bacillus show opposite trend $[9,10]$. Although Ilyonectria was generally described as the main causative agent for ginseng rusty root disease, a recent study revealed the potential association of the oxidation and deposition of rhizosphere $\mathrm{Fe}, \mathrm{Al}$, and $\mathrm{Mn}$ driven by nitrate-dependent $\mathrm{Fe}$ (II)-oxidizing bacteria with rusty root disease [11]. Moreover, the build-up of the Fusarium genus and accumulation of toxic diisobutyl phthalate (DiBP) is inversely associated with the population of Pseudomonas, Bacillus, and Burkholderia, etc., contributing to negative feedback between the soil and ginseng $[9,12]$. Similarly, the accumulation of phenolic acids in the rhizosphere of $R$. glutinosa potentially restrain the growth of Pseudomonas spp., but exert positive effects on mycelial growth, sporulation, and toxin production of Fusarium oxysporum [13]. Further research shows that the imbalance of beneficial (Pseudomonas spp.) and harmful (Enterobacter spp.) QS (quorum sensing) bacteria mediated by QQ (quorum quenching) bacteria in the rhizosphere, accounting for a key factor of $R$. glutinosa replant diseases [14].

A. sinensis is a typical alpine plant with high requirements for temperature $\left(20 \sim 24{ }^{\circ} \mathrm{C}\right)$, light (long daylight) and, high elevation zones $(2000 \sim 2500 \mathrm{~m})$. The production and quality of radix $A$. sinensis deteriorate following a decrease in altitude. Consequently, it becomes more vulnerable to early bolting, and this decreases the drug efficacy. The instability of the breeding base is the second largest reason affecting its quality. The traditional wasteland seedling raising manner that adopted all along was environmentally unsound. Due to the policy of Grain for Green and ecological protection, rotation of land seedling and cultivated wasteland seedling are regarded as the main choices. However, they are immature to guarantee the quality of $A$. sinensis. Reports on soil microbial communities of authentic $A$. sinensis are scanty due to the hurdles posed by the unique living environmental needs. Previously, we found that the number of dominant microbial species and the population diversities of bacteria, actinomyces, and fungi in the rhizosphere of Gansu province were more compared to other non-geoherb regions, including Yunnan and Sichuan province. Proteobacteria and bacteroidetes were the dominant 
bacterial phyla throughout the growing season of $A$. sinensis, whereas fungal dominant phyla varied with growth periods. Furthermore, the plant weight, root length, root diameter, soil $\mathrm{pH}$, rainfall, and climate temperature were the main factors contributing to the variation in microbial community composition of $A$. sinensis [15]. The most recent study on plant-microbe interactions mainly focused on succession cropping obstacles of medicinal plants, responses of plant-microbe to environmental stress, changes in rhizosphere nutrition conditions, root system biomass, and growth but provided little information on the accumulation of active constituents under different soil microenvironments [16]. There is an urgent need to elucidate the mechanism of the effects of rhizosphere microorganisms on the quality improvement of authentic $A$. sinensis, to acquire $A$. sinensis materials that is stable quality.

Herein, we prepared a pot experiment with four treatments: (i) GN-non-sterilized soil sampled from Gansu province, (ii) GS-sterilized soil sampled from Gansu province (iii) YN-non-sterilized soil sampled from Yunnan province, (iv) YS-sterilized soil sampled from Yunnan province, planting with the seedling variety of Gansu danggui 90-01. The four controls were without seedling implants. Through a combination of metabolomics and $16 \mathrm{~s}$ rRNA pyrosequencing technology, we aimed to explore (i) the potential variation of rhizosphere microorganisms, and the growth and quality of A. sinensis under different soil microenvironments, and (ii) establish whether the growth and quality of $A$. sinensis are positively impacted by changes in rhizosphere microorganisms.

\section{Results}

The morphology and quality of $A$. sinensis under different soil microenvironments

Regardless of whether the soil was sterilized or not, $A$. sinensis grown in Gansu soils (including GN and GS group) was significantly $(p<0.05)$ more branches and larger diameters than that grown in Yunnan soils (including YN and YS group) (Fig. 1A and B). Plants grown in sterilized soils displayed worse growth as indicated by the thinner rhizome, fewer number of branches and smaller diameter compared to those grown in non-sterilized soils (Fig. 1C and D). The survival rate of plants grown in non-sterilized soils $(\mathrm{GN}, 0.77 \pm 0.09$; YN, $0.63 \pm 0.18$; $n=6)$ was remarkably $(p<0.05)$ higher than that of plants grown in sterilized soils (GS, $0.48 \pm 0.15$; YS, $0.46 \pm 0.19 ; n=6)$. Further results showed that the average dry weight, root diameter, rootlet number, and root length of plants in GN group were significantly $(p<0.05)$ larger than that of plants of YN group. Plants of GS group
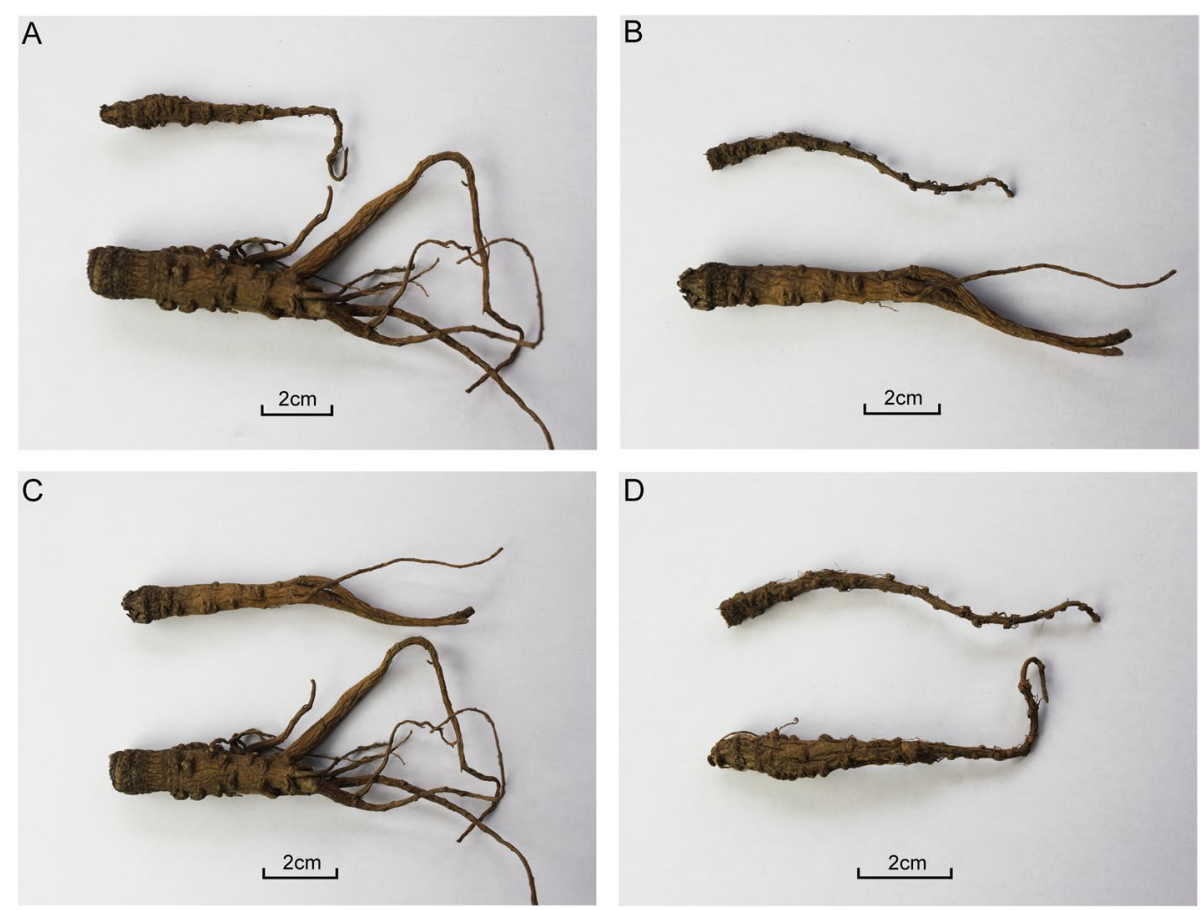

Fig. 1 Photographs of A. sinensis grown in different soil microenvironments. A: GN (below) and YN (above) sample; B: GS (below) and YS(above) sample; C: GN (below) and GS (above) sample; D: YN (below) and YS (above) sample. (Notice: GN represent Gansu non-sterilized group; YN represent Yunnan non-sterilized group; GS represent Gansu sterilized group; YS represent Yunnan sterilized group) 
Table 1 Root system growth of A. sinensis from different soil microenvironments

\begin{tabular}{|c|c|c|c|c|c|}
\hline Group & $\begin{array}{l}\text { Average surviving } \\
\text { rate } / \%\end{array}$ & Average dry weight /g & $\begin{array}{l}\text { Average root } \\
\text { diameter } / \mathrm{mm}\end{array}$ & Average rootlet number & Average root length $/ \mathrm{cm}$ \\
\hline GN & $0.77 \pm 0.09 a$ & $2.94 \pm 0.85 \mathrm{a}$ & $7.86 \pm 1.66 \mathrm{a}$ & $6.94 \pm 1.64 \mathrm{a}$ & $11.14 \pm 2.34 \mathrm{a}$ \\
\hline YN & $0.63 \pm 0.18 a$ & $0.88 \pm 0.25 b$ & $5.12 \pm 0.88 b$ & $1.72 \pm 0.80 b$ & $7.65 \pm 0.70 \mathrm{bc}$ \\
\hline GS & $0.48 \pm 0.15 b$ & $1.30 \pm 0.62 b$ & $6.14 \pm 1.13 b$ & $5.98 \pm 2.01 \mathrm{ab}$ & $10.36 \pm 1.13 c$ \\
\hline YS & $0.46 \pm 0.19 b$ & $0.36 \pm 0.05 b$ & $3.01 \pm 0.74 c$ & $1.00 \pm 0.00 b$ & $9.01 \pm 0.79 b$ \\
\hline
\end{tabular}

Values are mean \pm standard error $(n=6)$. Values with the same letter are not statistically signifificantly different, ANOVA, $p<0.05$

showed substantially $(p<0.05)$ reduced average dry weight, root diameter and root length. The root diameter of plants in YN group was significantly $(p<0.05)$ higher than in YS group, and higher in GS group than in YS group (Table 1).

To further explain the cause of differences in quality of $A$. sinensis among groups, medicinal components (phthalides and organic acids) and nutrients (amino acids, nucleosides and nucleobases) were compressively analyzed. The results showed that the contents of medicinal components were remarkably $(p<0.05)$ different among groups. Specifically, the level of chlorogenic acid and $Z$-ligustilide in $A$. sinensis grown on Yunnan soil was significantly $(p<0.05)$ higher than in plants grown on Gansu soil. The level of coniferyl ferulate and butylidenephthalide in the GN group was higher, whereas that of ferulic acid was lower than in other three groups. The level of senkyunolide A and butylphthalide in YN group was markedly $(p<0.05)$ higher than in the other groups. The level of senkyunolide I and senkyunolide H in YS group was significantly $(p<0.05)$ lower than in the other groups. However, the level of senkyunolide I and senkyunolide $\mathrm{H}$ was similar across GN, YN and GS groups (Table 2).

Previously, we found that $A$. sinensis possess high nutritive components including nucleosides, nucleobases and free amino acids [17]. The mean content of cytidine- $5^{\prime}$-monophosphate accounted for $31.03 \%$ of the total nucleosides and nucleobases, whereas adenosine accounted for $27.68 \%$, uridine for $17.84 \%$ and guanosine for $12.46 \%$, respectively, the mean content of arginine accounted for $86.91 \%$ of the total amino acids, whereas $\gamma$-aminobutyric acid accounted for $4.43 \%$, glutamine for $2.82 \%$ and proline for $0.88 \%$. The concentration of total nucleosides and nucleobases in YN group was significantly lower than in the other groups, where as that of essential amino acids such as lysine, tryptophan, valine in YN or YS group was lower than in GN or GS group. The differences in chemical composition across groups may be explained by differences in soil microenvironments (Supplementary Table S1).

\section{Variations in metabolites of $A$. sinensis under different soil microenvironments}

The quality of medicinal plants is influenced by the diversity and biochemistry of different compounds [18]. In this study, a simple, rapid and sensitive UPLC-UVQTOF-MS/MS method was developed to characterize metabolites in A. sinensis samples grown under different soil microenvironments. Principal components analysis (PCA) showed that the metabolic profiles varied significantly $(p<0.05)$ across groups, and the samples were clustered into four groups: GN, YN, GS and YS groups. Notably, GS and YS samples were clustered close to GN samples, especially GS samples. However, GN, GS and YS samples formed distant clusters from YN samples (Fig. 2A). The $R^{2} X, R^{2} Y$ and $Q^{2} Y$ of the OPLS-DA model were $0.667,0.966$ and 0.851 , respectively. The results of permutation analysis confirmed the stability and reliability of the OPLS-DA model. The hierarchical clustering heatmap showed that the molecular features within groups had similar clustering patterns, whereas

Table 2 Comparison of the analytes in different groups of $A$. sinensis samples $(\mathrm{mg} / \mathrm{g})$

\begin{tabular}{|c|c|c|c|c|c|c|c|c|c|}
\hline Group & $\begin{array}{l}\text { Chlorogenic } \\
\text { acid }\end{array}$ & Ferulic acid & Senkyunolide I & $\begin{array}{l}\text { Senkyunolide } \\
\mathrm{H}\end{array}$ & $\begin{array}{l}\text { Coniferyl } \\
\text { ferulate }\end{array}$ & $\begin{array}{l}\text { Senkyunolide } \\
\text { A }\end{array}$ & Butylphthalide & Z-ligustilide & butylidenephthalide \\
\hline GN & $1.89 \pm 0.15 c$ & $0.48 \pm 0.15 c$ & $0.22 \pm 0.09 a$ & $0.08 \pm 0.04 a$ & $0.51 \pm 0.42 \mathrm{a}$ & $0.02 \pm 0.03 b$ & - & $6.25 \pm 1.34 b$ & $0.35 \pm 0.08 \mathrm{a}$ \\
\hline YN & $3.62 \pm 0.35 a$ & $0.72 \pm 0.11 b$ & $0.18 \pm 0.05 a$ & $0.07 \pm 0.02 a$ & $0.08 \pm 0.04 b$ & $0.96 \pm 0.33 a$ & $0.35 \pm 0.26 a$ & $9.68 \pm 1.49 a$ & $0.23 \pm 0.06 b$ \\
\hline GS & $2.05 \pm 0.23 c$ & $0.87 \pm 0.07 a b$ & $0.23 \pm 0.09 a$ & $0.10 \pm 0.04 \mathrm{a}$ & $0.04 \pm 0.03 b$ & $0.07 \pm 0.09 b$ & - & $6.96 \pm 1.52 b$ & $0.26 \pm 0.09 b$ \\
\hline YS & $2.88 \pm 0.43 b$ & $0.97 \pm 0.24 \mathrm{a}$ & $0.04 \pm 0.00 b$ & $0.01 \pm 0.00 \mathrm{~b}$ & $0.10 \pm 0.07 b$ & $0.32 \pm 0.25 b$ & $0.03 \pm 0.04 b$ & $11.01 \pm 2.78 \mathrm{a}$ & $0.07 \pm 0.02 c$ \\
\hline
\end{tabular}

Values are mean \pm standard error $(n=6)$. Values with the same letter are not statistically signifificantly different, ANOVA, $p<0.05$

- Not detected 

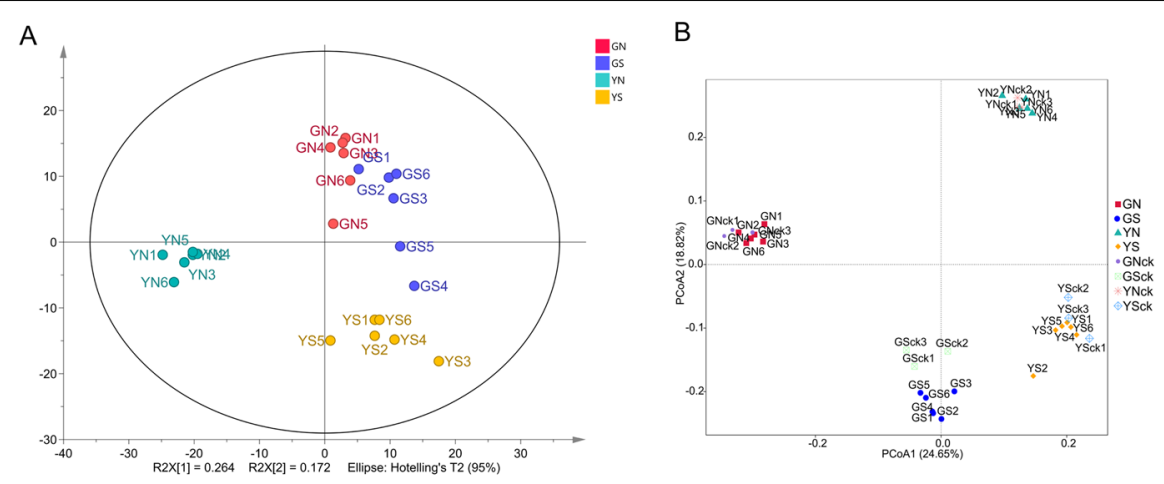

C
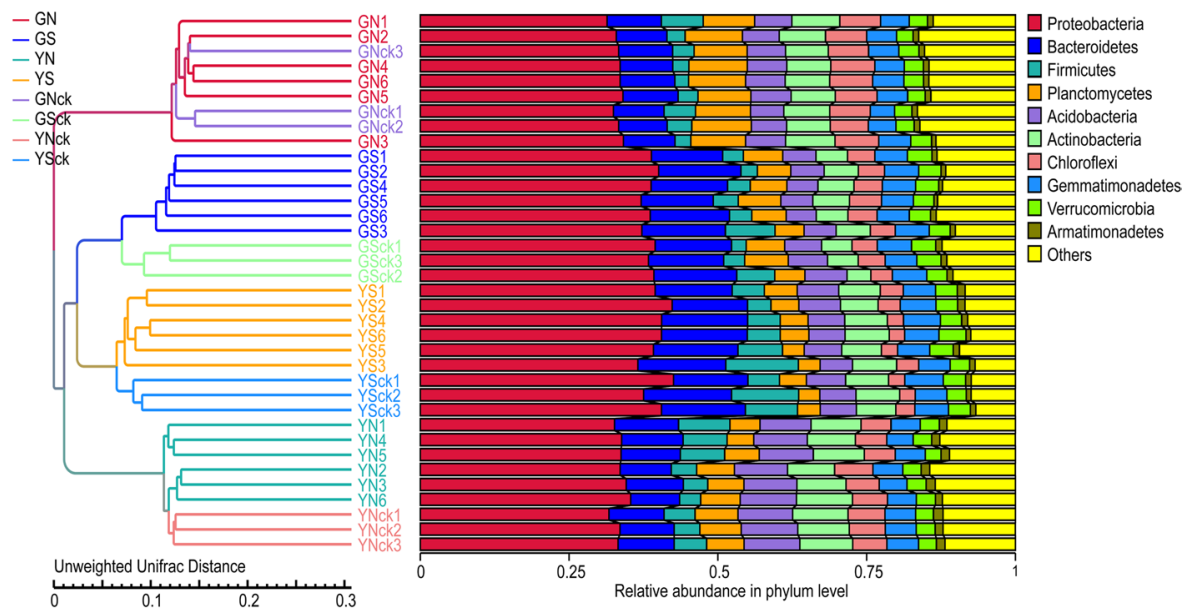

Fig. 2 The structure of metabolites and microbial communities of $A$. sinensis samples. PCA scores for the comparison of metabolomic profiles between GN, GS, YN, YS group (A); principal coordinates analysis (PCOA) based on unweighted UniFrac (UUF) distance metric (B); and unweighted pair-group method with arithmetic mean (UPGMA) clustering analyses at phylum level of samples from GN, GS, YN, YS, GNck, GSck, YNck, YSck group $(n=6)(\mathbf{C})$

molecular features between groups showed differences, which was consistent with the PCA clustering pattern (Fig. 3A).

In the UPLC-QTOF-MS/MS analysis, both positive and negative ion modes were utilized for qualitative identification. A total of 44 metabolites were identified by chemical standards or tentatively identified by comparing their quasi-molecular ions, empirical molecular formulas, and/or fragment ions with those of known chemicals (Supplementary Table S2) [19]. We selected markers that contributed greatly to the grouping by setting the Variable Importance for Projection (VIP) value greater than 1.5 and the $p$-value less than 0.05 in the Moderated t-Test. In general, the contents of ligustilide dimers was higher in plants of GN and GS groups, whereas the content of chlorogenic acid showed an opposite trend. More importantly, the plants of GN group had higher levels of ligustilide dimers, but lower levels of unknown [19], senkyunolide A and chlorogenic acid compared to YN group. Further analysis revealed that plants of GS group had higher levels of ligustilide dimers, Z-6,7-epoxyligustilide and senkyunolide I, while the level of chlorogenic acid and dicaffeoylquinic acid was lower compared to YS group. The results demonstrated that the plants in nonsterilized groups had higher levels of ligustilide dimers than those of the sterilized groups (Supplementary Fig. S3 and S2, Supplementary Table S3).

\section{Annotation and community composition of rhizosphere bacteria under different soil microenvironments}

The V4 region of $16 \mathrm{~S}$ rRNA gene in the total 36 samples was amplified using PCR and sequenced using the IonS5 ${ }^{\mathrm{TM}}$ XL platform. An average of 80,118 clean reads per sample were obtained. High-quality reads were clustered into 10,583 OTUs based on $>97 \%$ sequence identity, and then annotated using Silva132 database. A total of 65 OTUs were annotated at the phylum level, accounting for $87.60 \%$ of all OTUs; and 721 OTUs were annotated at the genus level. At the phylum level, we found that Proteobacteria (relative abundance of 55.38\%), Bacteroidetes 
A

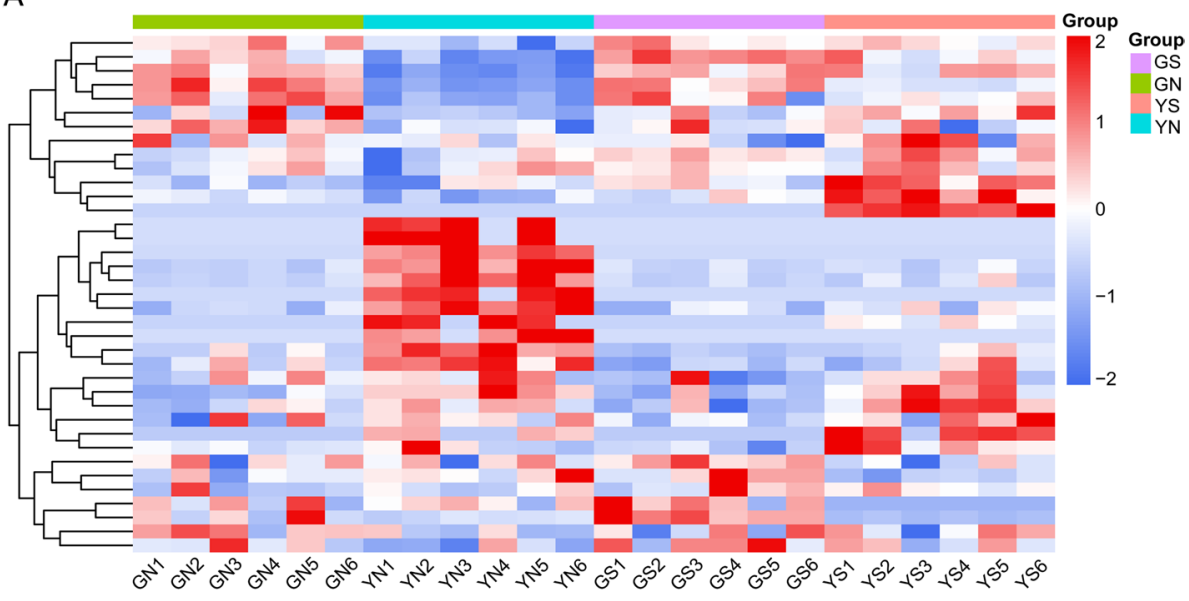

B
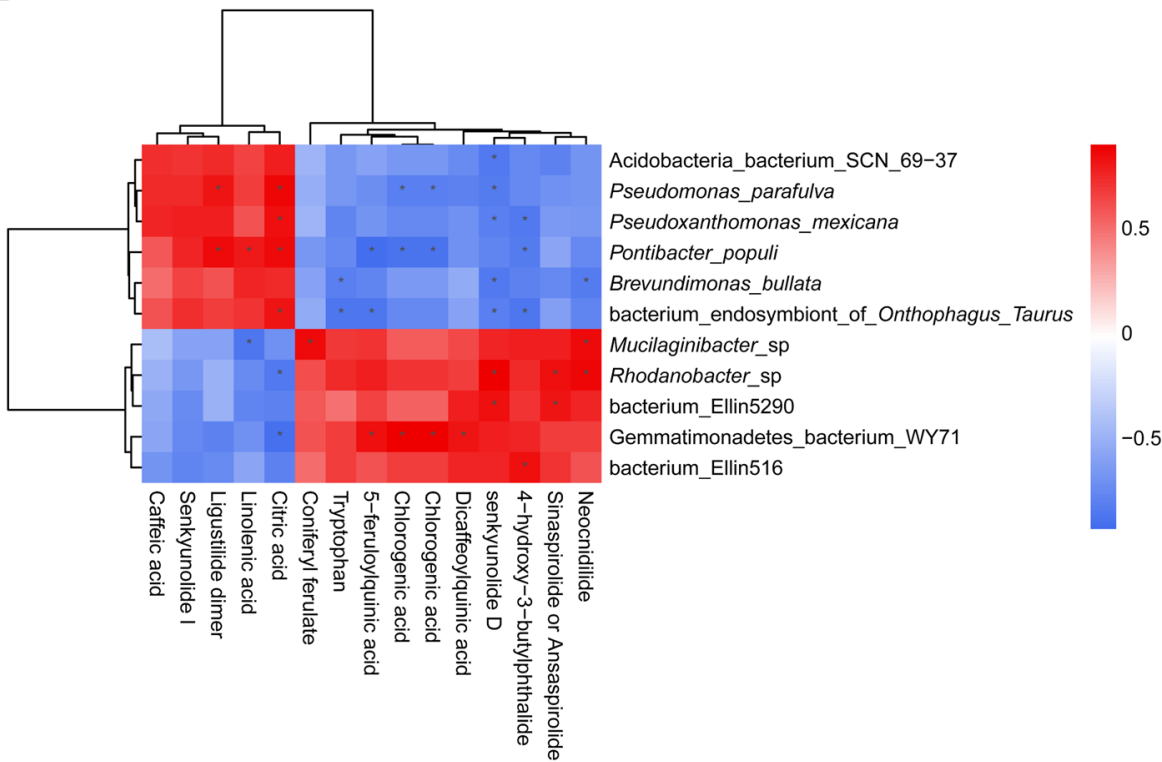

Fig. 3 Potentially mechanistic associations between rhizosphere microbes and metabolites. Hierarchical clustering heatmap shows a consistent clustering pattern within individual groups and a diverse clustering pattern between different groups (A); covariation between microbes and small molecules in A. sinensis, specifically those of differentially abundant microbes and metabolites matched against standards between GS and YS group (Spearman's rank correlation with two-tailed nominal $p$ values) $(n=6)(\mathbf{B})$

(10.65\%) and Acidobacteria (7.26\%) were the dominant species. At the genus level, the roots were dominated by Stenotrophomonas (9.97\%), Rhodanobacter (4.19\%) and Lactobacillus (1.48\%).

$\alpha$-diversity refers to the microbial diversity of a sample, similar conclusions were drawn by calculating the Chao1, Shannon's diversity index and Simpson diversity index, etc. (Fig. 4A and B). The results revealed higher community diversity in the non-sterilized groups than in the sterilized groups, and higher community diversity in the Gansu groups than in Yunnan groups. However, community diversity was not different between the planting groups and the corresponding non-planting groups. Results of ANOSIM showed that the difference between groups was significantly $(p<0.05)$ greater than that within groups, indicating that the results were reliable. Collectively, these results indicated that the diversity of the bacterial communities of $A$. sinensis was influenced by the soil microenvironments, and the plant effect had little influence.

PCoA based on weighted UniFrac (WUF) distance metric showed that the community composition of the planting groups and corresponding non-planting groups were highly similar. The sterilized and non-sterilized samples 


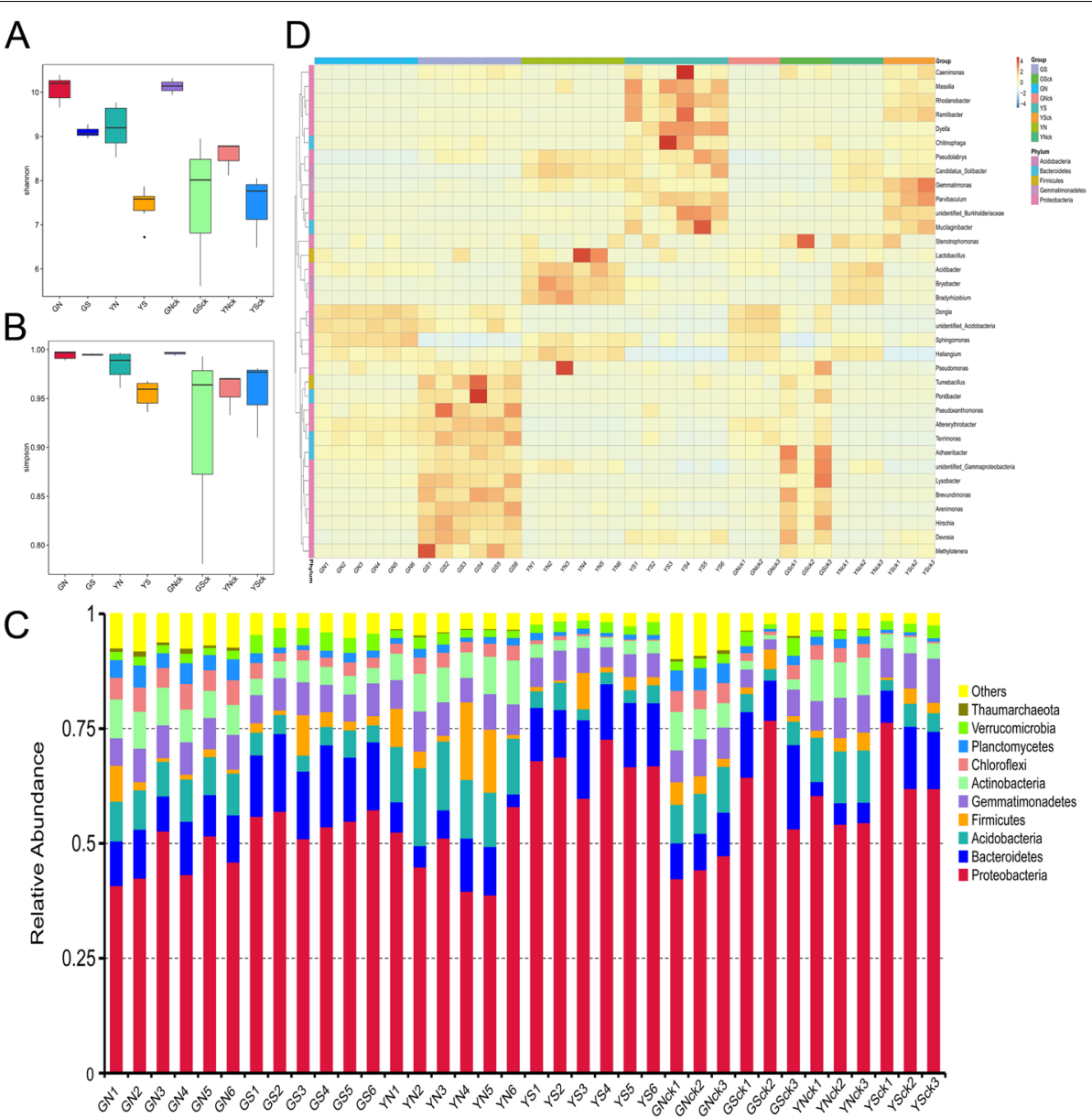

Fig. 4 The composition of bacteria rhizosphere microbial communities. Shannon index (A); simpson index (B); Phylum distribution (C); heatmap distribution and hierarchical clustering at the genus level (D) of samples from GN, GS, YN, YS, GNck, GSck, YNck, YSck group $(n=6)$

separated along the first axis, and PC1 explained 38.65\% of the total variation, indicating that indigenous bacteria were the most important factor affecting the rhizosphere bacterial community. Samples from Gansu and Yunnan were clearly distinguished on the second axis, and PC2 explained $23.35 \%$ of the total variation, indicating that geographical location were the second most important factor contributing to variations in rhizosphere bacterial community. However, when using an unweighted UniFrac (UUF) distance, geographic location showed largest contribution to the variation in the tested factors, and indigenous bacteria were the second most important source of variation (Fig. 2B).

The results of the PCoA were consistent with findings from unweighted pair-group method with arithmetic mean (UPGMA) clustering (Fig. 2C). Hierarchical clustering based on UUF distance metric showed complete clustering of samples in GN, GS, YN, and YS groups. However, it was observed that planting groups and the corresponding non-planting groups clustered together, indicating that plants had limited influence on the overall community composition. Samples from GS and YS initially clustered into one branch, then to YN samples, and finally the GN samples. This indicated that the GN group was different from the other three groups. To provide statistical support the above analysis, ADONIS and MRPP were employed to perform statistical tests on samples from different groups, and similar conclusion was reached.

\section{Comparison of composition of rhizosphere bacteria across groups}

To fully understand the distribution of OTU among groups, we constructed Venn diagrams, and used them to calculate the proportion of uniquely identified OTUs in each group and OTUs shared between different groups. It was found that the proportion of OTUs shared between GN and GNck groups, YN and YNck groups, GN and YN 
groups, GS and YS groups, GN and GS groups, YN and YS groups accounted for $48.74 \%, 42.17 \%, 38.17 \%, 30.10 \%$, $35.88 \%$, and $32.50 \%$ of all OTUs, respectively. This showed high similarity in terms of microbial community structure between the planting groups and corresponding nonplanting groups. A Venn diagram for all planting groups, showed that the number of unique OTUs belonging to the GN group was the highest, with 1692 species; and that of the YS group was the least, with 226 species. The same phenomena were recorded in all non-planting groups.

At the phylum level, the top 12 most abundant phylum from each sample or group were used to generate a accumulative column diagram showing the relative abundance of all species. The results showed that the microbial community was dominated by Proteobacteria, Bacteroidetes, and Acidobacteria. Moreover, a significantly $(p<0.05)$ higher relative abundance of Proteobacteria and Bacteroidetes, and a significantly $(p<0.05)$ lower relative abundance of Acidobacteria, Actinobacteria, and Chloroflexi was found in all sterilized groups compared to non-sterilized groups. The relative abundance of Chloroflexi and Planctomycetes was significantly $(p<0.05)$ higher in YN group, while that of Acidobacteria was significant $(p<0.05)$ lower than in GN group. In addition, the relative abundance of Gemmatimonadetes, Actinobacteria, and Chloroflexi was higher, while the relative abundance of Proteobacteria was lower in the GS group than in the YS group. The differences in microbial community between planting groups and corresponding non-planting groups was not significant $(p>0.05)$. Figure $4 \mathrm{C}$ shows the distribution of phylum level.

At the genus level, 721 taxa were identified from all 36 samples. The roots of $A$. sinensis were preferentially colonized by Stenotrophomonas, Rhodanobacter and Lactobacillus. A heatmap constructed based on hierarchical clustering of top 35 most abundant genera showed (Fig. 4D) that there were distinct differences in microbial community structure and relative abundance of most dominant genera across groups. Of note, the relative abundance of Lysobacter and Brevundimonas in Gansu group was significantly $(p<0.05)$ higher, whereas that of Rhodanobacter and Gemmatimonas was significantly $(p<0.05)$ lower than in Yunnan group. In addition, the relative abundance of Sphingomonas was higher in the GN group than in YN group, and the opposite was true for Mucilaginibacter. The relative abundance of Pseudomonas was higher in GS group than in YS group; while that of Ramlibacter and Sphingomonas showed opposite results. Of note, the relative abundance of Sphingomonas in all non-sterilized groups was significantly $(p<0.05)$ higher than in the corresponding sterilized groups.
Putative mechanisms regulating the relationship between microbes and quality-linked metabolites

The secondary metabolites of $A$. sinensis are mainly composed of phthalides, organic acids, and polysaccharides, among which phthalides are the main bioactive ingredients, with diverse structural features $[2,20]$. Previously, we found that Pseudomonas, Lysobacter, Pseudoxanthomonas, and Brevundimonas were significantly $(p<0.05)$ enriched in GS group than in the YS group. The contents of ligustilide dimer and senkyunolide I in GS group were significantly $(p<0.05)$ higher than in YS group, and were strongly positively correlated with $\mathrm{Pseu}$ domonas parafulva, Pseudoxanthomonas maxicana, and Brevundimonas bullata (average Spearman $\mathrm{r}>0.5$ ) (Fig. 3B). Among them, ligustilide dimer was extremely significantly $(p<0.05)$ positively correlated with $P$. parafulva (Spearman $\mathrm{r}=0.82$ ) (Supplementary Table S4). We therefore speculated that the high abundance of $P$. parafulva in the GS group contributed to the high levels of phthalides and was beneficial to the growth and quality improvement of $A$. sinensis.

Some ferulic acid derivatives such as ferulic acid ester have stronger biological activity and lower toxicity. In this study, organic acids and their esters were significantly $(p<0.05)$ enriched in YS group. This indicated that chlorogenic acid, dicaffeoylquinic acid and 5-feruloylquinic acid were positively associated with Gemmatimonadetes bacterium WY71 (average Spearman $\mathrm{r}>0.8$ ); coniferyl ferulate was positively associated with Mucilaginibacter sp. (Spearman $r=0.85$ ) (Fig. 3B) (Supplementary Table S4) [21]. Mucilaginibacter has been found to be abundant in the rhizosphere soil of Angelica medicinal plants. For example, $M$. herbaticus sp nov. [22], M. polysacchareus sp nov. [23], and $M$. angelicae sp nov [24]. were isolated from the rhizosphere, root surface of $A$. sinensis, and rhizosphere of A. polymorpha Maxim, respectively. We speculated that the high abundance of Mucilaginibacter in YS soil might be due to the high level of ferulic acid derivatives.

\section{Discussion}

Driving factors that determine the quality of $A$. sinensis under different soil conditions

Considerable reports indicate that the quality of Chinese drugs is significantly associated with geographical differences potentially attributed to the variation of altitude, climate, soil properties, and rhizospheric microorganisms, etc. Herein, we revealed that the soil microenvironment is a significant factor contributing to the quality improvement of $A$. sinensis. The tradition holds that higher the contents of ferulic acid and $Z$-ligustilide is associated with better quality of the radix A. Sinensis. Compared to the YN and GS groups, the GN 
group displayed significantly $(p<0.05)$ larger dry weight, root diameter, and root length (Table 1), whereas the contents of chlorogenic acid and $Z$-ligustilide in Yunnan groups were significantly $(p<0.05)$ higher than that of Gansu groups (Table 2). Moreover, the content of senkyunolide I, butylidenephthalide, ligustilide dimers were higher in Gansu groups compared to the Yunnan groups, whereas ferulic acid demonstrated a decreasing trend (Table 1, Supplementary Table S3). Zhang et al. found that the content of ferulic acid and levistolide $\mathrm{A}$ in non-geoherbs regions was higher than that of geoherbs regions, whereas senkyunolide I and butylidenephthalide demonstrated an opposite trend [19], which concur with the present conclusions. Contrarily, a large-scale field study found that the root length and head diameter showed a negative correlation with ferulic acid, and there was a negative association between root length and $Z$-ligustilide [25]. However, the evaluation of the quality of radix A.sinensis based on their appearance traits was inaccurate.

Phenolic acids have widely been reported as key allelochemicals of medicinal plants, including coumalic acid, ferulic acid, vanillic acid, 4-hydroxybenzoic acid, and syringic acid, which are found in root exudates of $P$. ginseng, R. glutinosa, and Pinellia ternata [26]. Imperatorin, vanillin, and ferulic acid are potential allelopathic autotoxic substances in rhizosphere soil and were found to significantly inhibit the growth of $A$. sinensis seedlings [27]. The high content of ferulic acid in plants of Yunnan groups indicates that these allelochemicals largely accumulate in the Yunnan soils, consequently deteriorating the growth of $A$. sinensis. Similarly, higher content of ferulic acid has been reported in sterilized groups than in non-sterilized groups, which may justify the weak growth of plants in the sterilized groups.

\section{Effect of soil type on the composition of rhizosphere bacteria of $A$. sinensis}

A continental-scale study on soil bacterial populations revealed that the richness and diversity of soil bacterial communities differ by ecosystem type, in particular, soil $\mathrm{pH}$ was the largest source of variation [28]. Compared to loessial soil and continuous cropping soil, polysaccharides and glucose amounts were significantly $(p<0.05)$ higher in Dioscorea opposita Thunb. growing in sandy soil. Moreover, the low soil $\mathrm{pH}$ was potentially associated with a high level of galacturonic acid from continuous cropping soil [29]. The soil of Gansu was slightly alkaline, whereas that of Yunnan was weakly acidic. Notably, variation in soil $\mathrm{pH}$ was attributed to significant differences in the rhizosphere and root-associated plant microbiomes. In the GN group, the relative abundance of Sphingomonas, Lysobacter, and Pseudoxanthomonas were higher than that of the YN group, whereas that of Rhodanobacter, Gemmatimonas, and Mucilaginibacter decreased significantly $(p<0.05)$ (Fig. 4D). Compared to the YS group, the relative abundance of Pseudomonas, Lysobacter and Pseudoxanthomonas were higher in the GS group, whereas that of Rhodanobacter, Ramlibacter, Dyella showed an opposite trend (Fig. 4D). Wu et al. found that the relative abundance of Lysobacter, Pseudoxanthomonas, Pseudomonas, and Burkholderia were significantly $(p<0.05)$ higher in the newly cultivated soil of $R$. glutinosa compared to continuous monocropping soil [30]. Similarly, the abundance of beneficial bacteria including Arthrobacter, Burkholderia, Rhodanobacter, and Sphingobacterium was negatively correlated with the accumulation of toxic DiBP in monocropping soil of $P$. ginseng [12]. Therefore, we deduced that the genus Lysobacter, Pseudomonas, and Burkholderia, etc. are potentially essential microbes for radix or rhizoma medicinal herbs.

Previously, Sphingomonas [31], Lysobacter [32, 33], and Pseudoxanthomonas [34, 35] were found to promote resistance to diseases and insect pests, as well as the degradation of harmful substances in the soil. Additionally, Sphingomonas promoted plant absorption through carbohydrate secretion into the rhizosphere [31]. Lysobacter strains exerted strong antagonistic activity against several pathogens, including Rhizoctonia solani, Fusarium oxysporum, and Xanthomonas carnpestris, etc.) [32]. Additional studies revealed that the genus Pseudomonas belongs to a group of plant growth-promoting rhizobacteria (PGPR) [36, 37]. For instance, Pseudomonas acted as a key PGPR in the rhizosphere of garlic regardless of growth periods, soil type, and agricultural practices. The constructed six Pseudomonas strains displayed strong plant growth-promoting effects [38]. These findings implied that stronger growth characteristics of $A$. sinensis in the Gansu group may be related to the high rhizosphere abundance of these antagonistic microorganisms.

\section{Effects of indigenous microorganisms on rhizosphere bacterial communities and growth of $A$. sinensis}

Many years of planting sole crops, including grain crops, vegetable crops, medicinal plants, etc., are frequently associated with widespread continuous cropping challenges. Speculation had it that soil sterilization could influence the physical structure and microbiological properties of soil. Subsequently, this triggered the nutrient release and inhibition of soil pathogens, thereby promoting plant growth. In the present study, A. sinensis from sterilized replant soil demonstrated higher plant performance and enhanced activities of active oxygen scavenging enzymes. Such effects may be driven by the increased number and diversity of culturable microbial 
populations and functional bacterial groups [39]. While soil sterilization could relieve the soil succession cropping failure, it eliminated indigenous microbes, including a few pathogens, and limited the potential beneficial effects of soil microbes to host plants [40]. Some reports show that indigenous microorganisms are critical in plant growth and agricultural productivity, and have potential benefits in plant secondary metabolism [41]. For instance, inoculation with PGPR Bacillus flexus alleviated damage from host salinity stress through modification of its physiological and biochemical state, for example, promoting photosynthesis, osmotic regulation, antioxidant enzymatic activities, and regulation of $\mathrm{Na}^{+}$/ $\mathrm{K}^{+}$homeostasis [42].

Herein, we found that plants grown in sterilized soil displayed worse growth and a low survival rate, which could be attributed to the newly cropped soil (Table 1). This validated the prediction that indigenous microorganisms are essential players in plant growth and development. Likewise, Glycyrrhiza uralensis grown in sterilized soil displayed poorer plant growth and photosynthesis. Inoculation with AM (Arbuscular mycorrhizae) fungi in sterilized soil compensated for the absence of indigenous microbial communities, which consequently promoted plant growth and increased glycyrrhizin and liquiritin contents [40]. Moreover, we found that the relative abundance of Sphingomonas was significantly $(p<0.05)$ higher in the GN group compared to the GS group, whereas Ramlibacter, Gemmatimonas, and Brevundimonas demonstrated an opposite trend (Fig. 4D). The relative abundance of Sphingomonas and Bradyrhizobium in the YN group was higher than that of the YS group, whereas Rhodanobacter, Ramlibacter, and Dyella were significantly $(p<0.05)$ lower (Fig. 4D). Additionally, Bradyrhizobium was revealed as a PGPR and could secrete plant hormones and QS metabolites, regulating the symbiotic relationship with the host and the adaptation to environmental variations [43]. QSdeficient (unable to produce cinnamoyl-HSL) mutant Bradyrhizobium sp. strains exhibited significantly $(p<0.05)$ lower colonization potential in rice roots and reduced plant growth compared to the wild-type strain [44]. The high abundance of beneficial bacteria, including Sphingomonas and Bradyrhizobium, in the rhizosphere of non-sterilized groups, accounted for better growth and quality of $A$. sinensis in the unsterilized groups.

\section{Potentially mechanistic association of rhizosphere microbes with metabolites}

Many plant endophytes can synthesize various secondary metabolites, some of them show good biological activities, which help to quality improvement of medicinal plants [45]. Inoculation with endophytes isolated from the alkaloid-rich genotype of Catharanthus roseus remarkably improves ajmalicine and serpentine contents in roots of low alkaloid yielding genotype [46]. The small molecules, including amino acids, organic acids, sugars, and secondary metabolites secreted by roots can promote the growth of some soil microorganisms, initiating the migration of soil microbial communities [47]. For instance, Falciphora oryzae colonized Arabidopsis roots through sensory signaling molecules derived from the roots. Subsequently, it could promote the lateral root growth of Arabidopsis through the biosynthesis of indole derivatives [48]. Therefore, we speculated that rhizosphere microbes may have a direct or indirect association with the growth and metabolism process of the host. Particularly, PGPR from Platycodon grandiflorum [49], mycorrhizal fungi from P. ginseng [50], and Actinomycetes from Curcuma longa [51] significantly promoted plant growth and resistance. Furthermore, the metabolite profiling of A. sinensis and microbial community composition in the rhizosphere soil exhibited similar variation patterns across groups. These observations implied that there may exist a specific correlation between the secondary metabolites of $A$. sinensis and its rhizosphere microorganisms.

Furthermore, our study found that higher contents of ligustilide dimers and senkyunolide I in the GS group demonstrated a positive correlation with $P$. parafulva, P. maxicana, and B. bullata (average Spearman $\mathrm{r}>0.5$ ). Higher contents of chlorogenic acid, dicaffeoylquinic acid, 5-feruloylquinic acid in the YS group were positively correlated with Gemmatimonadetes bacterium WY71 (average Spearman $r>0.8$ ). Similarly, positive association between coniferyl ferulate and Mucilaginibacter sp. (Spearman $\mathrm{r}=0.85$ ) (Supplementary Table S4). Of note, this positive association between a metabolite and species may denote that the metabolite promotes the growth of the species in question, or that the species produces that metabolite [52]. Studies have revealed that particular microorganisms can induce the accumulation of metabolites by (i) elevating the expression of genes associated with the synthesis of secondary metabolites, (ii) activating the host's defense responses, and (iii) synthesis of crucial enzymes that convert precursors into effective constituents [53]. A study found that Pseudomonas sp. and Pantoea sp. in the rhizosphere could stimulate the plant growth and synthesize of major phenolic acids in Salvia miltiorrhiza through its production of the abundant types and contents of phytohormones [54]. Elsewhere, inoculation of Trichoderma asperellum significantly increased the artemisinin concentration and dry weight 
of Artemisia апnиa L. leaves through elevated expression of artemisinin biosynthesis crucial enzymatic genes, HMGR1, FPS, ADS, CYP71AV1, CPR, DBR, $D X S 1$, and DXR1 [55]. Dark Septate Endophytes (DSE) could establish a symbiotic association with liquorice, increasing its plant $\mathrm{N}$ and $\mathrm{P}$ absorption, biomass, and accumulation of glycyrrhizic acid and glycyrrhizin [56]. The $P$. parafulva genome encod a series of antibacterial secondary metabolites, including lipopeptides, pyridine, benazine, and hydrogen cyanide, and exerts excellent antagonistic effects against rice and soybeans plant pathogens including Rhizoctonia solani, Xanthomonas axonopodis, and Burkholderia glumae [57, 58]. Further studies should explore whether the observed population structure stabilizes over time or is correlated with changes in metabolomic or microbiome composition in $A$. sinensis. Also, scholars should identify the plant genes associated with these taxa and extensively characterize their functions [7].

\section{Conclusion}

This study explored changes in metabolite profiling and rhizosphere microbial communities of $A$. sinensis under different soil microenvironments. It further provides a few insights into the mechanism by which rhizosphere microorganisms impact the quality improvement of authentic $A$. sinensis. The present findings affirm that the survival rate and growth status of A. sinensis in Gansu soils are better than that of Yunnan soils, higher in unsterilized groups than sterilized groups. High abundance of beneficial bacteria, including Sphingomonas, Pseudomonas, Lysobacter, Pseudoxanthomonas in Gansu soils and the enhanced growth characteristics of $A$. sinensis from Gansu soils demonstrated that the rhizosphere microbiome is an essential plant requirement for normal growth and development. Furthermore, ligustilide dimers enriched in the GS group demonstrated a notable positive correlation with the relative abundance of $P$. parafulva. Organic acids enriched in the YS group were positively correlated with the relative abundance of Gemmatimonadetes bacterium WY71 and Mucilaginibater sp. Collectively, these findings offer a foundation for further exploration of the relationship between rhizosphere microbes and the growth and quality formation of $A$. sinensis, and its mechanism. Such an approach is key in elucidating the mechanism of the association of microorganisms with the growth of medicinal plants.

\section{Materials and Methods Pot experiment site and design}

Soils for testing were sampled from the experimental base of the Min Conty Medicinal Plants Growing
Technology Extension Centre $\left(34^{\circ} 22^{\prime} 30^{\prime \prime} \mathrm{N}, 104^{\circ} 53^{\prime} 20^{\prime \prime}\right.$ $E ;$ black soil, $\mathrm{pH} \approx 8.0$ ) and the Medicinal Plants Research Institute Yunnan Academy of Agricultural Sciences $\left(26^{\circ} 28^{\prime} 42^{\prime \prime} \mathrm{N}, 100^{\circ} 4^{\prime} 34^{\prime \prime} \mathrm{E}\right.$; red soil, $\left.\mathrm{pH} \approx 6.5\right)$, whereby Vicia faba L. was the previously planted crop. The soil samples were transported to Shili Town of Min County, Gansu Province, with an average annual rainfall of $635.0 \pm 17.4 \mathrm{~mm}$, an annual average temperature of $5.43 \pm 0.71{ }^{\circ} \mathrm{C}$, and annual mean sunshine duration of $2154.3 \pm 68.7 \mathrm{~h}$. After sieving $(2 \mathrm{~mm})$, the soil samples were divided into two: One portion was sterilized by autoclaving at $121{ }^{\circ} \mathrm{C}$ for $4 \mathrm{~h}$, whereas the other portion was not sterilized. The $A$. sinensis seedlings (Gansu danggui 90-01) provided by Min County Xizhai town Zhucai herbs Planting Professional Cooperative, were transplanted during the local traditional planting period. We set the experiment into eight groups, including four experimental groups (GN, GS, YN, YS) and four control groups (GN-ck, GS-ck, YN-ck, YS-ck). The soil without seedling implant acted as a control group ( six repeats of each group).

The soil was put into a large plastic box and mixed thoroughly with a base fertilizer (diamine phosphate $10.6 \mathrm{~g}$ per pot, organic fertilizer $23.6 \mathrm{~g}$ per pot). The mixed soil was put to $4 \sim 6 \mathrm{~cm}$ lower the container mouth and compacted. Then, 8 seedlings were planted in each pot, with a depth of $3 \mathrm{~cm}$ and an interval of about $4 \mathrm{~cm}$. The seeds were covered with topsoil and a small amount of water (about $50 \mathrm{~mL}$ ) was applied to moisten the seeding layer. During the whole trial period, no fungicides were sprayed, manual weeding and pest control were timely completed. When the soil layer $2 \mathrm{~cm}$ below the topsoil dried, we irrigated each pot with the same amount of water. In 2018, A. sinensis plants were left to grow for 180 days. Carefully, the fresh plants were uprooted and gently shaken to remove loosely attached soil [30]. The rhizosphere soil tightly attached to tuberous roots was collected. Fresh plants and soil samples were transferred to the laboratory in an ice cooler and stored at $-80{ }^{\circ} \mathrm{C}$ awaiting analyses.

\section{DNA extraction, PCR amplification, and sequencing}

In total, 36 soil samples collected from the 8 groups of the pot experiment (GN, GS, YN, YS, GN-ck, GS-ck, YN-ck, YS-ck) were shipped to 100,015 (China) Beijing Novogene Biotech Co., Ltd. for 16S rRNA gene amplicon sequencing using the Ion $\mathrm{S}^{\mathrm{TM}} \mathrm{XL}$ platform. We employed the CTAB or SDS method to extract genomic DNA. To check for DNA purity and concentration, agarose gel electrophoresis was used. Then DNA was diluted with sterile water to $1 \mathrm{ng} \cdot \mu \mathrm{L}^{-1}$. The variable region $\mathrm{V} 4$ of the bacterial 16S rRNA gene was amplified using degenerate 
PCR primers $515 \mathrm{~F}$ and $806 \mathrm{R}$ (Walters et al. 2016). The samples were separated on a $2 \%(\mathrm{w} / \mathrm{v})$ agarose gel, and mixed in equivalent amounts based on the PCR product concentration. The mixed PCR products were purified using the GeneJET ${ }^{\mathrm{TM}}$ Gel Extraction Kit (Thermo Scientific). Briefly, 16S rRNA gene amplicons were extracted using Ion Plus Fragment Library Kit 48 rxns (Thermo Scientific) following the manufacturer's instructions. We sequenced the built DNA amplicon library on the Ion $\mathrm{S} 5^{\mathrm{TM}} \mathrm{XL}$ platform after Qubit quantification and examination of genomic libraries.

\section{Data processing and analysis}

The raw sequencing data from Ion AmpliSeq were processed using Cutadapt (V 1.9.1) (Martin M., 2011). The barcode and primer sequence for preliminary quality control were eliminated to obtain raw reads. To get clean reads, the chimera sequences were detected and deleted from the dataset using the UCHIME algorithm. The clean reads were clustered into operational taxonomic units (OTUs) at a sequence similarity level of 97\% using Uparse software (v7.0.1001). Taxonomic annotation via the SSUrRNA database was performed using Mothur (version 1.25.1) and SILVA132 (http:// www.arb-silva.de/). The MUSCLE (V 3.8.31) software was employed to perform multiple sequence alignment to establish the phylogenetic relationship of all OTUs sequences. The QIIME (V 1.9.1) and R software (V 2.15.3) were applied for sample diversity analysis. $\alpha$-diversity was evaluated considering the OTU tables. Spearman rank correlation analysis was conducted in $\mathrm{R}$ using the vegan packages.

\section{A multi-index comprehensive evaluation of the chemical composition of $A$. sinensis \\ Method 1: Preparation of sample solutions for secondary metabolite analysis}

Exactly $0.2 \mathrm{~g}$ dried powder (40 mesh) was sonicated $\left(100 \mathrm{~Hz}, 25^{\circ} \mathrm{C}\right)$ with $4 \mathrm{~mL} 70 \%$ methanol for $45 \mathrm{~min}$ in a $50 \mathrm{~mL}$ glass-stoppered conical flask. The samples were centrifuged $(12,000 \mathrm{r} / \mathrm{min}, 10 \mathrm{~min})$. The supernatants were injected directly onto an A Thermo Syncronis $\mathrm{C}_{18}$ $(2.1 \mathrm{~mm} \times 100 \mathrm{~mm}, 1.7 \mu \mathrm{m})$ column. The mobile phase constituted A ( $0.1 \%$ formic acid) and B (acetonitrile). The optimized elution conditions included: $0 \sim 2 \mathrm{~min}$, $5 \% \sim 10 \% \mathrm{~B} ; 2 \sim 10 \mathrm{~min}, 10 \% \sim 40 \% \mathrm{~B} ; 10 \sim 14 \mathrm{~min}$, $40 \% \sim 58 \% \mathrm{~B} ; 14 \sim 20 \mathrm{~min}, 58 \% \sim 90 \%$ B; $20 \sim 22 \mathrm{~min}$, 90\% 90\% B; $22 \sim 22.5 \mathrm{~min}, 90 \% \sim 5 \% \mathrm{~B} ; 22.5 \sim 24 \mathrm{~min}$, $5 \% \sim 5 \% \mathrm{~B}$. The flow rate was $0.4 \mathrm{~mL} \cdot \mathrm{min}^{-1}$, column temperature was kept at $35{ }^{\circ} \mathrm{C}$, with was $2 \mu \mathrm{L}$ injection volume. Mass spectrometric detection was performed on a Waters ACQUITY ${ }^{\mathrm{TM}}$ Synapt Q-TOF mass spectrometer equipped with an electrospray ionization
(ESI) source. ESI-MS spectra were generated in both positive $\left(\mathrm{ESI}^{+}\right)$and negative $\left(\mathrm{ESI}^{-}\right)$ion modes through scanning from 100 to $1000 \mathrm{Da}$. The optimized conditions are referenced to published articles in Chemistry Central Journal [59].

\section{Method 2: Preparation of nucleosides, nucleobases, and amino acids analysis samples and standard solutions for qualitative and quantitative analyses}

Referenced to published articles in Molecules [17]. A mixed standard stock solution of 28 reference compounds was prepared in methanol/water $(9: 1, \mathrm{v} / \mathrm{v})$, with concentrations as follows: 2'-deoxyguanosine $3.6667 \mu \mathrm{g} \cdot \mathrm{mL}^{-1}$, cytidine $4.3750 \mu \mathrm{g} \cdot \mathrm{mL}^{-1}, 2^{\prime}$ - deoxycytidine $4.9167 \mu \mathrm{g} \cdot \mathrm{mL}^{-1}$, cytidine-5'-monophosphate $4.1250 \mu \mathrm{g} \cdot \mathrm{mL}^{-1}, \quad 2^{\prime}$-deoxyadenosine $3.9167 \mu \mathrm{g} \cdot \mathrm{mL}^{-1}$, adenosine $4.3750 \mu \mathrm{g} \cdot \mathrm{mL}^{-1}$, guanosine $4.0833 \mu \mathrm{g} \cdot \mathrm{mL}^{-1}$, inosine $3.5417 \mu \mathrm{g} \cdot \mathrm{mL}^{-1}$, uridine $5.2083 \mu \mathrm{g} \cdot \mathrm{mL}^{-1}$, ornithine $3.6333 \mu \mathrm{g} \cdot \mathrm{mL}^{-1}$, leucine $15.0000 \mu \mathrm{g} \cdot \mathrm{mL}^{-1}$, iso-leucine $15.0000 \mu \mathrm{g} \cdot \mathrm{mL}^{-1}$, phenylalamine $15.3333 \mu \mathrm{g} \cdot \mathrm{mL}^{-1}$, tryptophan $15.8333 \mu \mathrm{g} \cdot \mathrm{mL}^{-1}$, aminobutyric acid $19.0000 \mu \mathrm{g} \cdot \mathrm{mL}^{-1}$, methionine $17.0000 \mu \mathrm{g} \cdot \mathrm{mL}^{-1}$, proline $18.5000 \mu \mathrm{g} \cdot \mathrm{mL}^{-1}$, valine $17.1667 \mu \mathrm{g} \cdot \mathrm{mL}^{-1}$, trans4-hydroxy-L-proline $21.0000 \mu \mathrm{g} \cdot \mathrm{mL}^{-1}$, glutamincacid $15.6667 \mu \mathrm{g} \cdot \mathrm{mL}^{-1}$, glutamine $13.8333 \mu \mathrm{g} \cdot \mathrm{mL}^{-1}$, asparagine $23.8333 \mu \mathrm{g} \cdot \mathrm{mL}^{-1}$, tyrosine $15.3333 \mu \mathrm{g} \cdot \mathrm{mL}^{-1}$, threonine $17.3333 \mu \mathrm{g} \cdot \mathrm{mL}^{-1}$, lysine $18.5000 \mu \mathrm{g} \cdot \mathrm{mL}^{-1}$, serine $15.5000 \mu \mathrm{g} \cdot \mathrm{mL}^{-1}$, citrulline $17.3333 \mu \mathrm{g} \cdot \mathrm{mL}^{-1}$, and arginine $32.3333 \mu \mathrm{g} \cdot \mathrm{mL}^{-1}$. The working standard solution for the calibration curves were prepared by diluting the mixed standard solution with $10 \%$ methanol at different concentrations.

\section{Method 3: Preparation of organic acids and phthalides analysis samples and mixed control solutions}

Here, $0.2 \mathrm{~g}$ dried powder (40 mesh) was extracted in the ultrasound-assisted condition at $25{ }^{\circ} \mathrm{C}$ with $20 \mathrm{~mL}$ methanol for $40 \mathrm{~min}$ in a $50 \mathrm{~mL}$ glass-stoppered conical flask. After centrifugation $(13,000 \mathrm{r} / \mathrm{min}, 10 \mathrm{~min})$ and filtration $(0.22 \mu \mathrm{m}$ membrane filter $)$, the supernatants were injected into the UPLC system for analysis. A mixed standard stock solution of 9 reference compounds was prepared in methanol/water $(9: 1, \mathrm{v} / \mathrm{v})$, with concentrations as follows: chlorogenic acid $24.1313 \mathrm{mg} \cdot \mathrm{L}^{-1}$, ferulic acid $15.8838 \mathrm{mg} \cdot \mathrm{L}^{-1}$, senkyunolide $\mathrm{I} 7.5600 \mathrm{mg} \cdot \mathrm{L}^{-1}$, senkyunolide $\mathrm{H} 3.0208 \mathrm{mg} \cdot \mathrm{L}^{-1}$, coniferyl ferulate $18.0340 \mathrm{mg} \cdot \mathrm{L}^{-1}$, senkyunolide A $5.3025 \mathrm{mg} \cdot \mathrm{L}^{-1}$, n-butylphthalide $199.9788 \mathrm{mg} \cdot \mathrm{L}^{-1}$, Z-ligustilide $17.9196 \mathrm{mg} \cdot \mathrm{L}^{-1}$, butylidenephthalide $15.0 \mathrm{mg} \cdot \mathrm{L}^{-1}$. The working standard solution for calibration curves was prepared by diluting the mixed standard solution with methanol at different concentrations. The separation was done on a Thermo Syncronis $\mathrm{C}_{18}(2.1 \mathrm{~mm} \times 100 \mathrm{~mm}, 1.7 \mu \mathrm{m})$ column. The 
mobile phase constituted: A ( $0.1 \%$ formic acid) and B (acetonitrile) with a gradient elution: $0 \sim 2 \mathrm{~min}, 5 \% \sim 10 \%$ B; $2 \sim 10 \mathrm{~min}, 10 \% \sim 40 \% \mathrm{~B} ; 10 \sim 13 \mathrm{~min}, 40 \% \sim 40 \% \mathrm{~B}$; $13 \sim 19$ min, $40 \% \sim 50.8 \%$ B; $19 \sim 20$ min, $50.8 \% \sim 90 \%$ B; $20 \sim 21 \mathrm{~min}, 90 \% \sim 90 \% \mathrm{~B} ; 21 \sim 23 \mathrm{~min}, 90 \% \sim 5 \%$ B. Column temperature was maintained at $35{ }^{\circ} \mathrm{C}$; the flow rate was $0.4 \mathrm{~mL} \cdot \mathrm{min}^{-1}$, with a detection wavelength of $260 \mathrm{~nm}, 280 \mathrm{~nm}, 320 \mathrm{~nm}$ and injection volume of $2 \mu \mathrm{L}$. Linear regression models between sample concentration and peak area were established to generate linear regression equation and linear ranges of chlorogenic acid, ferulic acid, senkyunolide I, senkyunolide $\mathrm{H}$, coniferyl ferulate, senkyunolide A, n-butylphthalide, Z-ligustilide, and butylidenephthalide respectively.

\section{Statistical analysis}

All statistical analyses were performed in R software (V 2.15.3) (R Foundation for Statistical Computing, Vienna, Austria) and IBM SPSS 20.0 (IBM Corporation, USA). Analysis of variance (ANOVA) and multiple comparison analysis was applied to calculate the mean and standard deviation and for statistical tests. The differences of parameter variance were estimated by ANOVA and the Kruskal Wallis rank-sum test based on the distribution of parameter statistics. For post-hoc comparison, Tukey's honest significant differences tests and Wilcoxon matched-pairs signed-rank test were applied. R (V 3.2.2) was employed to perform heat map, PCoA, and hierarchical clustering. The $\mathrm{R}$ language did a comparison of microorganisms through the 'adonis' function from R's 'vegan' package. The multiple null hypothesis testing of the prior group was achieved using the 'anosim' or 'adonis' function from R's 'vegan' package. According to the method of Franzosa et al. [52], we clustered the differentially abundant features via a custom approach. Then, the correlation analysis of the differentially abundant chemical components with the differentially abundant microorganisms was undertaken (For all clustering analyses, we applied Spearman's rank correlation as a similarity measure with a threshold of $r=0.7)$. Unless otherwise stated, the significance level was set at $p<0.05$.

\footnotetext{
Abbreviations

TCM: Traditional Chinese medicine; DiBP: Diisobutyl phthalate; QS: Quorum sensing; QQ: Quorum quenching; UPLC-UV-QTOF-MS/MS: Ultra Performance Liquid Chromatography_UItra Violet_Quadrupole Time of Flight_Mass/ Mass; OPLS-DA: Orthogonal partial least-squares discrimination analysis; PCA: Principal components analysis; VIP: Variable importance for projection; OTU: Operational taxonomic units; ANOSIM: Analysis of similarities; PCOA: Principal coordinates analysis; WUF: Weighted UniFrac; UUF: Unweighted UniFrac; UPGMA: Unweighted pair-group method with arithmetic mean; ADONIS: Permutational multivariate analysis of variance; MRPP: Multi Response Permutation Procedure; PGPR: Plant growth-promoting rhizobacteria; AM: Arbuscular mycorrhizae; DSE: Dark Septate Endophytes; ANOVA: Analysis of varia.
}

\section{Supplementary Information}

The online version contains supplementary material available at https://doi. org/10.1186/s12870-021-03047-w.

Additional file 1: Table S1. Comparison of the nucleosides, nucleobases, and essential amino acids contents in different groups of $A$. sinensis samples.

Additional file 2: Table S2. Secondary metabolites identified in A. sinensis by untargeted metabolomics.

Additional file 3: Table S3. Tentative markers for discriminating A. sinensis samples between every two groups. Note: A, GN and YN group; B, GS and YS group; C, GN and GS group; D, YN and YS group. Figure S1. OPLS-DA scores (A), permutation Test of OPLS-DA model (B), S-Plot (C), and VIP value (D) for the comparison of metabolomic profiles between $G N$ and $Y N$ group in positive ion mode. Figure S2. OPLS-DA scores (A), permutation Test of OPLS-DA model (B), S-Plot (C), and VIP value (D) for the comparison of metabolomic profiles between GS and YS group in positive ion mode.

Additional file 4: Table S4. Spearman's rank correlation scores of representative metabolites and representative species between GS and YS group.

\section{Acknowledgements}

We thank Tang YP for useful suggestions, thank Guo ZX for assistance with the seedling samples and granting permission to conduct research in Min Conty Angelica sinensis institute, and we thank our laboratory coworkers from Key Laboratory of Monitoring and Management of Crop Diseases and Pest Insects for their technical support.

\section{Authors' contributions}

Zhu $\mathrm{L}$ and Yan $\mathrm{H}$ participated in the design of the study. Zhu $\mathrm{L}$ carried out the experiments, data acquisition, and drafted the manuscript. Yan $\mathrm{H}$, Zhou GS, Jiang $\mathrm{CH}$ also contributed to figures generation, literature search, and manuscript editing. Liu P, Yu G performed the statistical data analysis. Guo S, Wu QN, Duan JA contributed to the discussion of the manuscript. All authors read and approved the final manuscript.

\section{Funding}

This work was supported by National Natural Science Foundation of China (No. 81773848), Ministry of Finance Central Level of the Special (No. 2060302), 2019 Foshan-HKUST Fund (FSUST19-SRI10), and Special Technology System Project for the Modern Agricultural Industry (CARS-21).

\section{Availability of data and materials}

The datasets used and/or analysed during the current study are available from the corresponding author on reasonable request.

\section{Declarations}

Ethics approval and consent to participate Not applicable.

Consent for publication

Not applicable.

\section{Competing interests}

The authors declare that they have no competing interests.

\section{Author details}

${ }^{1}$ National and Local Collaborative Engineering Center of Chinese Medicinal Resources Industrialization and Formulae Innovative Medicine, Jiangsu Collaborative Innovation Center of Chinese Medicinal Resources Industrialization, State Administration of Traditional Chinese Medicine Key Laboratory of Chinese Medicinal Resources Recycling Utilization, Nanjing University of Chinese Medicine, Nanjing, China. ${ }^{2}$ State Key Laboratory of Crop Genetics and Germplasm Enhancement, Nanjing Agricultural University, Nanjing, China. 
Received: 23 December 2020 Accepted: 11 May 2021

Published online: 22 June 2021

\section{References}

1. Hook ILI. Danggui to Angelica sinensis root: Are potential benefits to European women lost in translation? A review. J Ethnopharmacol. 2014;152(1):1-13.

2. Wei WL, Zeng R, Gu CM, Qu Y, Huang LF. Angelica sinensis in China-A review of botanical profile, ethnopharmacology, phytochemistry and chemical analysis. J Ethnopharmacol. 2016;190:116-41.

3. Berendsen RL, Pieterse CMJ, Bakker PAHM. The rhizosphere microbiome and plant health. Trends Plant Sci. 2012;17(8):478-86.

4. Mendes R, Kruijt M, De Bruijn I, Dekkers E, Van Der Voort M, Schneider $J H M$, et al. Deciphering the rhizosphere microbiome for disease-suppressive bacteria. Science. 2011;332(6033):1097-100.

5. Bulgarelli D, Rott M, Schlaeppi K, van Themaat EV , Ahmadinejad N, Assenza F, et al. Revealing structure and assembly cues for Arabidopsis root-inhabiting bacterial microbiota. Nature. 2012;488(7409):91-5.

6. Edwards J, Johnson C, Santos-Medellín C, Lurie E, Podishetty NK, Bhatnagar $S$, et al. Structure, variation, and assembly of the root-associated microbiomes of rice. Proc Natl Acad Sci U S A. 2015;112(8):E911-920.

7. Walters WA, Jin Z, Youngblut N, Wallace JG, Sutter J, Zhang W, et al. Large-scale replicated field study of maize rhizosphere identifies heritable microbes. Proc Natl Acad Sci USA. 2018;115(28):7368-737.

8. Lakshmanan V, Selvaraj G, Bais HP. Functional soil microbiome: Belowground solutions to an aboveground problem. Plant Physiol. 2014;166(2):689-700.

9. Luo L, Guo C, Wang L, Zhang J, Deng L, Luo K, et al. Negative Plant-Soil Feedback Driven by Re-assemblage of the Rhizosphere Microbiome With the Growth of Panax notoginseng. Front Microbiol. 2019;10:1597.

10. Wu L, Chen J, Khan MU, Wang J, Wu H, Xiao Z, et al. Rhizosphere fungal community dynamics associated with Rehmannia glutinosa replant disease in a consecutive monoculture regime. Phytopathology. 2018;108(12):1493-500.

11. Wei X, Wang X, Cao P, Gao Z, Chen AJ, Han J. Microbial Community Changes in the Rhizosphere Soil of Healthy and Rusty Panax ginseng and Discovery of Pivotal Fungal Genera Associated with Rusty Roots. Biomed Res Int. 2020;1:1-13.

12. Dong L, Xu J, Zhang L, Cheng R, Wei G, Su H, et al. Rhizospheric microbial communities are driven by Panax ginseng at different growth stages and biocontrol bacteria alleviates replanting mortality. Acta Pharm Sin B. 2018;8(2):272-82.

13. Wu L, Wang J, Huang W, Wu H, Chen J, Yang Y, et al. Plant-microbe rhizosphere interactions mediated by Rehmannia glutinosa root exudates under consecutive monoculture. Sci Rep. 2015;5:15871.

14. Li Q, Wu Y, Wang J, Yang B, Chen J, Wu H, et al. Linking Short-Chain N-Acyl Homoserine Lactone-Mediated Quorum Sensing and Replant Disease: A Case Study of Rehmannia glutinosa. Front Plant Sci. 2020;11:87.

15. An Z, Guo F, Chen Y, Bai G, Chen Z. Rhizosphere bacterial and fungal communities during the growth of Angelica sinensis seedlings cultivated in an Alpine uncultivated meadow soil. Peer J. 2020;8:e8541.

16. Canarini A, Kaiser C, Merchant A, Richter A, Wanek W. Root exudation of primary metabolites: Mechanisms and their roles in plant responses to environmental stimuli. Front Plant Sci. 2019;10:157.

17. Zhu S, Guo S, Duan JA, Qian D, Yan H, Sha X, et al. UHPLC-TQ-MS coupled with multivariate statistical analysis to characterize nucleosides, nucleobases and amino acids in Angelicae Sinensis Radix obtained by different drying methods. Molecules. 2017;22(6):918.

18. De Vos RCH, Moco S, Lommen A, Keurentjes JJB, Bino RJ, Hall RD. Untargeted large-scale plant metabolomics using liquid chromatography coupled to mass spectrometry. Nat Protoc. 2007;2(4):778-91.

19. Zhang K, Yan M, Han S, Cong L, Wang L, Zhang L, et al. Identification of Chemical Markers for the Discrimination of Radix Angelica sinensis Grown in Geoherb and Non-Geoherb Regions Using UHPLC-QTOF-MS/MS Based Metabolomics. Molecules. 2019;24(19):3536.

20. Zou J, Chen GD, Zhao H, Wang XX, Zhang ZJ, Qu YB, et al. Triangeliphthalides A-D: Bioactive phthalide trimers with new skeletons from: Angelica sinensis and their production mechanism. Chem Commun. 2019;55(44):6221-4.
21. Ma JP, Guo ZB, Jin L, Li YD. Phytochemical progress made in investigations of Angelica sinensis (Oliv.) Diels. Chin J Nat Med. 2015;13(4):241-9.

22. Lee HR, Han SI, Rhee KH, Whang KS. Mucilaginibacter herbaticus sp. nov., isolated from the rhizosphere of the medicinal plant Angelica sinensis. Int J Syst Evol Microbiol. 2013;63(Pt 8):2787-93.

23. Han SI, Lee HJ, Lee HR, Kim KK, Whang KS. Mucilaginibacter polysacchareus sp. nov., an exopolysaccharide-producing bacterial species isolated from the rhizoplane of the herb Angelica sinensis. Int J Syst Evol Microbiol. 2012:62(Pt 3):632-7.

24. Kim BC, Poo H, Lee KH, Kim MN, Kwon OY, Shin KS. Mucilaginibacter angelicae sp. nov., isolated from the rhizosphere of Angelica polymorpha maxim. Int J Syst Evol Microbiol. 2011;62(Pt 1):55-60.

25. Giacomelli N, Yongping Y, Huber F, Ankli A, Weckerle C. Angelica sinensis (Oliv.) Diels: Influence of Value Chain on Quality Criteria and Marker Compounds Ferulic Acid and Z-Ligustilide. Medicines. 2017;4(1):14.

26. Zhang B, Weston PA, Gu L, Zhang B, Li M, Wang F, et al. Identification of phytotoxic metabolites released from Rehmannia glutinosa suggest their importance in the formation of its replant problem. Plant Soil. 2019;441(1-2):439-54.

27. Xin A, Li $X$, Jin $H$, Yang $X$, Zhao $R$, Liu J, et al. The accumulation of reactive oxygen species in root tips caused by autotoxic allelochemicals - A significant factor for replant problem of Angelica sinensis (Oliv.) Diels. Ind Crops Prod. 2019;138:111432.

28. Fierer N, Jackson RB. The diversity and biogeography of soil bacterial communities. Proc Natl Acad Sci USA. 2006;103(3):626-31.

29. Ma F, Wang R, Zhu J, Zhang Y, Wang Y, Hu W, et al. Characterisation comparison of polysaccharides from Dioscorea opposita Thunb. growing in sandy soil, loessial soil and continuous cropping. Int J Biol Macromol. 2019;126:776-85.

30. Wu L, Wang J, Wu H, Chen J, Xiao Z, Qin X, et al. Comparative metagenomic analysis of rhizosphere microbial community composition and functional potentials under Rehmannia glutinosa consecutive monoculture. Int J Mol Sci. 2018;19(8):2394.

31. Takeuchi M, Sakane T, Yanagi M, Yamasato K, Hamana K, Yokota A. Taxonomic study of bacteria isolated from plants: Proposal of Sphingomonas rosa sp. nov., Sphingomonas pruni sp. nov., Sphingomonas asaccharolytica sp. nov., and Sphingomonas mali sp. nov. Int J Syst Bacteriol. 1995:45(2):334-41.

32. Expósito RG, Postma J, Raaijmakers JM, De Bruijn I. Diversity and activity of Lysobacter species from disease suppressive soils. Front Microbiol. 2015;6:1243.

33. De Bruijn I, Cheng $X$, de Jager $V$, Expósito RG, Watrous J, Patel N, et al. Comparative genomics and metabolic profiling of the genus Lysobacter. BMC Genomics. 2015;16:991.

34. Kumar M, Revathi K, Khanna S. Biodegradation of cellulosic and lignocellulosic waste by Pseudoxanthomonas sp R-28. Carbohydr Polym. 2015:134:761-6.

35. Patel V, Cheturvedula S, Madamwar D. Phenanthrene degradation by Pseudoxanthomonas sp. DMVP2 isolated from hydrocarbon contaminated sediment of Amlakhadi canal, Gujarat, India. J Hazard Mater. 2012;201-202:43-51.

36. Silby MW, Winstanley C, Godfrey SAC, Levy SB, Jackson RW. Pseudomonas genomes: Diverse and adaptable. FEMS Microbiol Rev. 2011;35(4):652-80.

37. Oteino N, Lally RD, Kiwanuka S, Lloyd A, Ryan D, Germaine KJ, et al. Plant growth promotion induced by phosphate solubilizing endophytic Pseudomonas isolates. Front Microbiol. 2015;6:745.

38. Zhuang L, Li Y, Wang Z, Yu Y, Zhang N, Yang C, et al. Synthetic community with six Pseudomonas strains screened from garlic rhizosphere microbiome promotes plant growth. Microb Biotechnol. 2020;14(2):488-502.

39. Zhang XH, Lang DY, Zhang EH. Effects of soil sterilization on growth of Angelica sinensis plant and soil microbial populations in a continuous mono-cropping soil. Int J Agric Biol. 2016;18(2):458-63.

40. Yu M, Xie W, Zhang X, Zhang S, Wang Y, Hao Z, et al. Arbuscular mycorrhizal fungi can compensate for the loss of indigenous microbial communities to support the growth of liquorice (Glycyrrhiza uralensis fisch.). Plants. 2020;9(1):7.

41. Castrillo G, Teixeira PJPL, Paredes SH, Law TF, De Lorenzo L, Feltcher ME, et al. Root microbiota drive direct integration of phosphate stress and immunity. Nature. 2017;543(7646):513-8. 
42. Xiong YW, Li XW, Wang TT, Gong Y, Zhang CM, Xing K, et al. Root exudates-driven rhizosphere recruitment of the plant growth-promoting rhizobacterium Bacillus flexus KLBMP 4941 and its growth-promoting effect on the coastal halophyte Limonium sinense under salt stress. Ecotoxicol Environ Saf. 2020;194:110374.

43. Bogino PC, Nievas FL, Giordano W. A review: Quorum sensing in Bradyrhizobium. Appl Soil Ecol. 2015;94:49-58.

44. Cai W, Ou F, Staehelin C, Dai W. Bradyrhizobium sp. strain ORS278 promotes rice growth and its quorum sensing system is required for optimal root colonization. Environ Microbiol Rep. 2020;12(6):656-66.

45. Nicoletti R, Ferranti P, Caira S, Misso G, Castellano M, Di Lorenzo G, et al. Myrtucommulone production by a strain of Neofusicoccum australe endophytic in myrtle (Myrtus communis). World J Microbiol Biotechnol. 2014;30(3):1047-52.

46. Singh S, Pandey SS, Shanker K, Kalra A. Endophytes enhance the production of root alkaloids ajmalicine and serpentine by modulating the terpenoid indole alkaloid pathway in Catharanthus roseus roots. J Appl Microbiol. 2020;128(4):1128-42.

47. Hartmann A, Schmid M, van Tuinen D, Berg G. Plant-driven selection of microbes. Plant Soil. 2009;321(1-2):235-57.

48. Sun X, Wang N, Li P, Jiang Z, Liu X, Wang M, et al. Endophytic fungus Falciphora oryzae promotes lateral root growth by producing indole derivatives after sensing plant signals. Plant Cell Environ. 2020;42(2):358-73.

49. Huang CM, Chen WC, Lin SH, Wang YN, Shen FT. Exploration of rootassociated bacteria from the medicinal plant Platycodon grandiflorum Microbes Environ. 2019;34(4):413-20.

50. Kim YS, Balaraju K, Jeon YH. Biological characteristics of Bacillus amyloliquefaciens AK-0 and suppression of ginseng root rot caused by Cylindrocarpon destructans. J Appl Microbiol. 2017;122(1):166-79.

51. Nakaew N, Lumyong S, Sloan WT, Sungthong R. Bioactivities and genome insights of a thermotolerant antibiotics-producing Streptomyces sp. TM32 reveal its potentials for novel drug discovery. Microbiologyopen. 2019;8(11):1-7.
52. Franzosa EA, Sirota-Madi A, Avila-Pacheco J, Fornelos N, Haiser HJ, Reinker $\mathrm{S}$, et al. Gut microbiome structure and metabolic activity in inflammatory bowel disease. Nat Microbiol. 2019;4(2):293-305.

53. Zhai X, Jia M, Chen L, Zheng CJ, Rahman K, Han T, et al. The regulatory mechanism of fungal elicitor-induced secondary metabolite biosynthesis in medical plants. Crit Rev Microbiol. 2017;43(2):238-61.

54. You H, Yang S, Zhang L, Hu X, Li O. Promotion of phenolic compounds production in Salvia miltiorrhiza hairy roots by six strains of rhizosphere bacteria. Eng Life Sci. 2018;18(3):160-8.

55. Zhai T, Wang Y, Liu C, Liu Z, Zhao M, Chang Y, et al. Trichoderma asperellum ACCC30536 inoculation improves soil nutrition and leaf artemisinin production in Artemisia annua. Acta Physiol Plant. 2019;41(4):1-11.

56. He C, Wang W, Hou J. Characterization of dark septate endophytic fungi and improve the performance of liquorice under organic residue treatment. Front Microbiol. 2019;10:1364.

57. Zhang Y, Chen P, Ye G, Lin H, Ren D, Guo L, et al. Complete Genome Sequence of Pseudomonas Parafulva PRS09-11288, a Biocontrol Strain Produces the Antibiotic Phenazine-1-carboxylic Acid. Curr Microbiol. 2019;76(9):1087-91.

58. Kakembo D, Lee YH. Analysis of traits for biocontrol performance of Pseudomonas parafulva JBCS1880 against bacterial pustule in soybean plants. Biol Control. 2019;134:72-81.

\section{Publisher's Note}

Springer Nature remains neutral with regard to jurisdictional claims in published maps and institutional affiliations.
Ready to submit your research? Choose BMC and benefit from:

- fast, convenient online submission

- thorough peer review by experienced researchers in your field

- rapid publication on acceptance

- support for research data, including large and complex data types

- gold Open Access which fosters wider collaboration and increased citations

- maximum visibility for your research: over 100M website views per year

At BMC, research is always in progress.

Learn more biomedcentral.com/submissions 